\title{
DIMENSIONAMENTO DE PESSOAL DE ENFERMAGEM EM UMA \\ UNIDADE ESPECIALIZADA EM TRANSPLANTE DE FÍGADO: \\ COMPARAÇÃO DO REAL COM O PRECONIZADO
}

\section{THE CALCULATION OF THE NUMBER OF THE NURSING STAFF IN A LIVER TRANSPLANT UNIT: A COMPARISON BETWEEN PRESCRIBED PROCEDURES AND ACTUAL SITUATIONS}

\author{
Maria Aparecida de Abreu Tanos* \\ Maria Cristina Komatsu Braga Massarollo** \\ Raquel Rapone Gaidzinski***
}

TANOS, M.A.A. et al. Dimensionamento de pessoal de enfermagem em uma unidade especializada em transplante de fígado: comparação do real com o preconizado. Rev.Esc.Enf.USP., v.34, n.4, p. 376-82, dez. 2000.

\begin{abstract}
RESUMO
O estudo foi desenvolvido em uma clínica cirúrgica de um hospital público universitário, onde, dentre as modalidades terapêuticas oferecidas, são realizados transplantes de fígado. Nessa clinica são internados pacientes que necessitam de cuidados de enfermagem de diferentes complexidades, desde intermediários até os intensivos. Teve-se como objetivo comparar o quadro de pessoal de enfermagem existente na unidade coin o preconizado pela Resolução COFEN $n^{\circ} 189 / 96$, onde ficou demonstrado que o quadro da unidade respeita essa Resolução.
\end{abstract}

PALAVRAS-CHAVE: Pessoal de enfermagem. Sistema de classificação de pacientes. ABSTRACT

The study was developed in a surgical clinic of a public-university hospital where, among the offered therapeutic modalities, they are accomplished, also, liver transplants. In that clinic they are interned patient that, need caring of nurs

ing of different complexities, from the intermediate from to the intensive ones. The objective was to compare the staff nursing existents in the unit for the installment of nursing attendance with the preconized for the Resolution COFEN $n^{\circ} 189 / 96$. It's conclude that the existent staff nursing respects the preconized for this Resolution.

KEYWORDS: Staff nursing. System of patient classification.

\section{INTRODUÇÃO}

O dimensionamento de recursos humanos de enfermagem é a primeira etapa para provimento de pessoal e tem por objetivo a previsão do número de funcionários por categoria, necessária para atender, direta ou indiretamente, às necessidades de assistência de enfermagem da clientela (KURCGANT et a1.12).

Uma das funções do enfermeiro responsável pelo gerenciamento é o suprimento dos recursos humanos para a prestação da assistência, o que lhe proporciona inúmeras dificuldades, decorrentes de motivos econômicos, políticos e dependentes dos objetivos da instituição.

Para RIBEIRO16, a previsão de pessoas para as atividades de enfermagem nos hospitais é um problema que se baseia, fundamentalmente, na determinação da categoria profissional que a instituição se dispõe a contratar e na definição de suas funções, o que, por sua vez, depende da filosofia e da política institucional, bem como, do conceito que a direção tem da enfermagem.

\footnotetext{
1 fermeira - Departamento de Enfermagem do Hospital Universitário da USP.

** Enfermeira - Professora Doutora do Departamento de Orientação Profissional da Escola de Enfermagem da USP.

*** Enfermeira - Professora Associada do Departamento de Orientação Profissional da Escola de Enfermagem da USP.
} 
KURCGANT et a1.12 e MAGALHÃES et al. ${ }^{14}$, também, citam que a caracterização da instituição e do serviço de enfermagem, quanto à filosofia, objetivos e propostas assistenciais, deve ser o ponto de partida para a previsão de pessoal.

O dimensionamento inadequado dos recursos humanos em enfermagem traz implicações sobre o resultado da qualidade da assistência de enfermagem prestada à clientela, em virtude dos aspectos quantitativos e qualitativos de pessoal estarem diretamente ligados ao produto final do seu trabalho, que é a qualidade da assistência prestada ao paciente.

Segundo GAIDZINSKI ${ }^{9}$, exaustivos estudos destinados a calcular o pessoal de enfermagem têm sido desenvolvidos ao longo dos anos na tentativa de se estabelecer um método que se ajuste às reais necessidades dos diversos serviços. A contagem do número de leitos ocupados, a proporcionalidade de pessoal de enfermagem e a relação paciente e hora média de assistência de enfermagem têm sido experimentados como parâmetros para o dimensionamento do pessoal de enfermagem. Contudo, esses métodos tradicionais têm se mostrado insatisfatórios por considerarem todos os pacientes como detentores do mesmo nível de atenção de enfermagem, não levando em conta a gravidade dos mesmos e a sua conseqüente influência na alocação de recursos humanos na unidade".

Para GIOVANNETTI11, "dimensionar recursos humanos para a assistência de enfermagem pode se tornar mais racional e efetivo quando se procura agrupar pacientes em categorias que reflitam a magnitude do processo de cuidar".

Considerando que dimensionar recursos humanos em enfermagem está vinculado ao tipo de cuidado necessário a cada paciente, tem sido proposto utilizar como critério, no dimensionamento pessoal de enfermagem, o Sistema de Classificação de Pacientes (SCP) baseado no Cuidado Progressivo ao Paciente (CPP). O CPP, como critério para dimensionar o pessoal de enfermagem, foi introduzido no Brasil por RIBEIRO 16, em 1972, embora tenha iniciado, de forma empírica, desde os tempos de Florence Nightingale, que buscava localizar, mais convenientemente na enfermaria, os pacientes que demandavam maior atenção de enfermagem.

RIBEIRO 16, em seu estudo, apontou o conceito de CPP como um método para instrumentalizar o dimensionamento de recursos humanos em enfermagem, com a finalidade de assegurar uma distribuição mais eqüitativa da assistência e aumentar a produtividade e eficiência hospitalar. Estabelecido o conceito de CPP, inicia-se de forma sistematizada a classificação dos pacientes por tipo de cuidado. O SCP é definido como o processo pelo qual se procura categorizar pacientes de acordo com a quantidade de cuidado de enfermagem requerido, ou seja, baseado na complexidade da assistência de enfermagem.

RODRIGUES FILHO 17 enfatizou a importância da utilização do SCP para o processo decisório em relação ao dimensionamento de recursos humanos em enfermagem, recomendando a inclusão deste tema nas áreas de pesquisa em enfermagem no Brasil.

Pesquisas sobre o dimensionamento de pessoal de enfermagem utilizando o SCP foram desenvolvidos por ALCALA et al.', ALVES et al. 2, CAMPEDELLI et al. 4, FUGULIN; GAIDZINSKI 7, GAIDZINSKI ${ }^{8}$, LIMA ${ }^{13}$ e SANTOS ${ }^{18}$.

O SCP considera o número médio de horas despendidas pela equipe de enfermagem, segundo cada categoria de cuidado. Os parâmetros do SCP diferem de um modelo para outro e em nenhum deles são explicadas as condições em que esses parâmetros foram determinados.

A falta de parâmetros bem definidos, destinados à operacionalização do dimensionamento de pessoal de enfermagem, faz com que este assunto se torne polêmico, causando falta de argumentação para as chefias de enfermagem diante da administração do hospital. Isso possibilita a interferência de profissionais de outras áreas na determinação da quantidade e qualidade do pessoal de enfermagem quando a competência para o dimensionamento de recursos humanos em enfermagem deve ser dos enfermeiros que atuam diretamente na assistência (CAMPEDELLI et al. ${ }^{4} \mathrm{e}$ GAIDZINSKI9).

Diante da inexistência de uma regulamentação sobre critérios mínimos para dimensionar o pessoal de enfermagem, o COFEN ${ }^{6}$, em sua Resolução n¹89/ 96, estabeleceu parâmetros para dimensionamento do quadro de profissionais de enfermagem nas instituições de saúde. Segundo essa Resolução, o cálculo de pessoal de enfermagem é competência do enfermeiro, que deve observar o SCP e considerar as horas de assistência de enfermagem distribuidas em percentuais, de acordo com a categoria profissional, nos diferentes tipos de cuidados: minimo ou auto-cuidado, intermediário, semi-intensivo e intensivo.

Conforme diferencia-se o cuidado de enfermagem a cada paciente, varia o número de horas de enfermagem despendidas ao mesmo e, conseqüentemente, variando também, a quantidade e a qualificação do pessoal de enfermagem envolvido.

Considerando a relevância deste tema para o gerenciamento em enfermagem decidiu-se pela realização deste estudo. 


\section{OBJETIVOS}

- Dimensionar o quadro de pessoal de enfermagem da unidade, segundo o preconizado pela Resolução COFEN n¹89/966;

- Correlacionar o quadro de pessoal existente na unidade com o preconizado pela Resolução COFEN n¹89/966.

\section{MÉTODO}

Por se tratar de um caso particular, optou-se pelo estudo de caso. Segundo CHIZzOTTI5, o estudo de caso caracteriza-se por designar as pesquisas que coletam e registram dados de um caso particular ou de vários casos, a fim de organizar um relatório ordenado e crítico de uma experiência.

\section{Local do Estudo}

Esse estudo foi desenvolvido em uma clínica de um hospital público universitário geral, especializada no tratamento de doenças hepatobiliares, onde, dentre as modalidades terapêuticas oferecidas, são realizados, também, transplantes de figado.

Essa clínica é constituída por 19 leitos, distribuídos em 6 quartos com 2 leitos cada um, outros 2 com um leito cada um, destinados a pacientes conveniados e 3 UTIs, sendo duas UTIs com 2 leitos e uma UTI com 1 leito.

O quadro do pessoal de enfermagem é composto por 16 enfermeiros, sendo 1 enfermeira chefe, 3 enfermeiros encarregados, 12 enfermeiros assistenciais, 21 auxiliares de enfermagem e 2 atendentes de enfermagem, distribuídos em 4 turnos de trabalho: manhã, tarde, noite par e noite impar, com uma carga horária de trabalho de $40 \mathrm{~h}$ semanais cada um.

\section{População}

Nessa clínica são internados pacientes de diferentes graus de complexidade assistencial. A população desse estudo foi constituída por todos os pacientes internados no período de 20/10/97 a 10/11/97.

\section{Procedimento de Coleta de Dados}

Após autorização da instituição para a realização do estudo, foram contatadas a diretora da área e a enfermeira chefe da unidade, para exposição dos objetivos do trabalho, esclarecimento quanto ao método e instrumentos utilizados e a programação do período e horário da coleta de dados.
Para proceder ao dimensionamento de pessoal de enfermagem, a fim de projetar o quadro, segundo os parâmetros de horas de assistência de enfermagem da Resolução COFEN n¹89/966, utilizou-se o modelo desenvolvido por GAIDZINSKI 8 que recomenda as seguintes fases:

\section{Conhecimento do grau de complexidade assistencial dos pacientes.}

Optou-se pelo instrumento de PERROCA 15 para classificação dos pacientes, por adequar-se mais às características da clínica. Esse instrumento avalia 13 indicadores: estado mental e nivel de consciência, oxigenação, sinais vitais, nutrição e hidratação, motilidade, locomoção, cuidado corporal, eliminações, terapêutica, educação à saúde, comportamento, comunicação e integridade cutânea

O instrumento foi testado durante 3 dias. Logo após este período, foi aplicado em todos os pacientes internados, durante 16 dias, no horário de 10 às $11 \mathrm{~h}$ da manhã, de segunda a sexta-feira.

Para a aplicação do instrumento era consultado o censo diário, para a verificação dos pacientes internados na clínica e os respectivos números dos leitos. Após, eram colhidos os dados para preenchimento do instrumento de classificação dos pacientes e feita a classificação do grau de dependência de cada um.

\section{Horas de assistência de enfermagem.}

Foram adotados os parâmetros da Resolução COFEN n¹89/96', que discorre em seu artigo $4^{\circ}$ que, para efeito de cálculo, "devem ser consideradas como horas de Enfermagem por leito, nas 24 horas:

- 3 horas de enfermagem, por cliente, na assistência minima ou auto-cuidado;

- 4,9 horas de enfermagem, por cliente, na assistência intermediária;

- 8,5 horas de enfermagem, por cliente, na assistência semi-intensiva;

- 15,4 horas de enfermagem, por cliente, na assistência intensiva".

Essa Resolução preconiza, também, a seguinte proporção de horas de assistência por categoria profissional:

- $27 \%$ de enfermeiros e $73 \%$ de técnicos/auxiliares de enfermagem - cuidados mínimos e intermediários;

- 40\% de enfermeiros e $60 \%$ de técnicos/auxiliares de enfermagem - cuidados semi-intensivos;

- 55,6\% de enfermeiros e 44,4\% de técnicos/auxiliares de enfermagem - cuidados intensivos. 


\section{Conhecimento do percentual de ausências previstas e não previstas.}

Considerou-se como ausências previstas os dias relativos às folgas (descanso remunerado semanal e feriados), e às férias. Como ausências não previstas, o dias relativos às faltas, às licenças e às suspensões.

Para prever pessoal de enfermagem para a cobertura dessas ausências, foram calculados os índices a partir da proposição de GAIDZINSKI 8:

- folgas - para cobertura de 2 folgas semanais: 40\%;

- feriados - para cobertura de cerca de 13 feriados/ ano, não coincidentes com o domingo: 4\%;

- férias - para cobertura de 30 dias de férias/ano: 9\%;

- absenteísmo - para cobertura de faltas e licenças em hospitais públicos, enfermeiro, 15 dias/ano: $4 \%$ e auxiliar de enfermagem, 20 dias/ano: 6\%.

\section{Aplicação da equação para dimensionar o pessoal}

\section{de enfermagem}

A partir de variáveis levantadas, nas fases anteriores, procedeu-se ao cálculo do pessoal de enfermagem, aplicando-se a seguinte equação:

$$
q=\sum_{k} \frac{P_{k}}{100} \cdot \frac{\sum_{j}\left(n_{j} \cdot h_{j}\right)}{t \cdot p} \cdot\left[\left(1+\frac{e}{d-e}\right) \cdot\left(1+\frac{f}{D-f}\right) \cdot\left(1+\frac{v_{k}}{D-v_{k}}\right) \cdot\left(1+\frac{a_{k}}{D-a_{k}}\right)\right]
$$

Onde

$\mathrm{q}$ = quantidade total de pessoas de enfermagem;

$\mathrm{Pk}$ proporção percentual da categoria $\mathrm{k} ; \mathrm{k}=$ categoria profissional;

$\mathrm{nj}$ = quantidade média diária de pacientes que necessitam do tipo de cuidado $\mathrm{j}$;

$\mathrm{j}$ = tipo de cuidado requerido pelo paciente (mínimo, intermediário, semi-intensivo, intensivo);

h. = tempo médio de cuidado de cada paciente que necessita do cuidado j;

$\mathrm{t}=$ tempo diário de trabalho de cada profissional dado em horas, na unidade em estudo corresponde, em média a 8 horas; $\mathrm{p}=$ produtividade média $(80 \%$ do tempo diário de trabalho, dedicado ao cuidado do paciente);

$\mathrm{e}=$ número de folgas semanais que necessitam de cobertura (no caso, 2 por semana);

$\mathrm{f}=$ número de dias feriados por ano, não coincidentes com o domingo;

$\mathrm{v}_{\mathrm{k}}=$ número de dias de férias por ano, por categoria profissional;

ak = número médio de dias de ausências não previstas (absenteísmo) por categoria profissional, por ano;

$\mathrm{d}=$ dias de funcionamento da unidade (no caso, 7

dias);

$\mathrm{D}=$ dias trabalhados no ano (no caso, 365 dias);

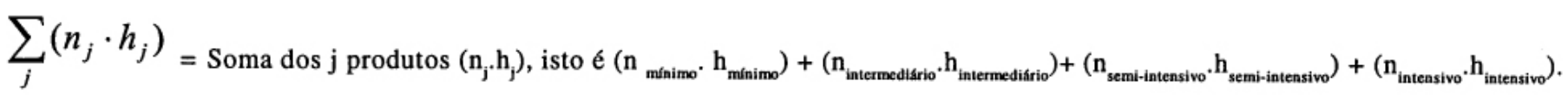

Para comparação do quadro preconizado com o quadro existente, partiu-se do quadro de pessoal da unidade, subtraindo-se o índice para cobertura das ausências previstas e não previstas, tomando-se como base os dados da Divisão de Enfermagem, referentes ao período de janeiro a dezembro de 1996. Foram consideradas como faltas, as ausências por faltas de qualquer tipo (abonada, justificada e injustificada). As licenças: médica, maternidade e acidente de trabalho foram estudadas individualmente, no entanto, agrupamos como outras licenças as referentes a nojo, gala e paternidade, devido ao baixo índice de ocorrência desses tipos de ausências não previstas.

Esses índices foram calculados a partir da seguinte equação proposta por GAIDZINSKI 8:

$$
A_{f} \%=\left(\frac{N_{f}}{D F-N_{f}}\right) \cdot 10
$$

Onde:

$\mathrm{A}_{f} \%=$ percentual de um dado tipo de ausência $f$; por categoria profissional

$N_{I}=$ soma dos dias de um dado tipo de ausência $f$ ocorrida na unidade durante o, período de um ano, por categoria profissional;

$F=$ número de funcionários da categoria profissional em estudo (enfermeira, auxiliar de enfermagem, etc); $D$ = dias de funcionamento da unidade durante o ano ( $D$ = 365 dias/ano, no caso de unidades estudadas onde o funcionamento é ininterrupto).

Os valores obtidos estão expressos no Quadro 1. 
QUADRO 1- Demonstrativo das ausências previstas e não previstas em uma unidade especializada em transplante hepático, período de jan. a dez./96.

\begin{tabular}{|c|c|c|c|c|c|c|c|c|c|c|c|c|}
\hline \multirow{3}{*}{$\begin{array}{c}\text { CATEGORIA } \\
\text { PROFIS- } \\
\text { SIONAL }\end{array}$} & \multirow{3}{*}{$\begin{array}{c}\text { QTDE. } \\
\text { MEDIA DE } \\
\text { PESSOAL }\end{array}$} & \multicolumn{3}{|c|}{ AUSÊNCIAS PREVISTAS } & \multicolumn{7}{|c|}{ AUSÊNCIAS NÃO PREVISTAS } & \multirow[b]{3}{*}{ TOTAL } \\
\hline & & & & & \multicolumn{5}{|c|}{ LICENÇAS } & \multirow{2}{*}{$\begin{array}{l}S \\
U \\
S \\
p \\
E \\
N \\
C C \\
\text { Ô } \\
E \\
S\end{array}$} & \multirow{2}{*}{$\begin{array}{l}A \\
B \\
S \\
E \\
N \\
T \\
E \\
I \\
S \\
M \\
O\end{array}$} & \\
\hline & & $\begin{array}{l}\mathbf{O} \\
\mathbf{L} \\
\mathbf{G} \\
\mathbf{A} \\
\mathrm{S}\end{array}$ & $\begin{array}{c}\text { É } \\
\text { R } \\
\text { I } \\
\text { A } \\
\text { S }\end{array}$ & $\begin{array}{l}S \\
O \\
M \\
A\end{array}$ & $\begin{array}{l}A \\
L \\
T \\
A \\
S\end{array}$ & $\begin{array}{l}\text { M } \\
\text { É } \\
\text { D } \\
1 \\
\text { C } \\
\text { A }\end{array}$ & $\begin{array}{l}M \\
A \\
T \\
E \\
R\end{array}$ & $\begin{array}{l}A \\
C \\
i \\
D \\
E \\
N \\
T \\
E\end{array}$ & $\begin{array}{l}\mathrm{I} \\
\mathrm{N} \\
\mathrm{S}\end{array}$ & & & \\
\hline \multirow{2}{*}{ ENFERMEIRO } & \multirow[t]{2}{*}{10,00} & 1130 & 299 & 1429 & 26 & 34 & 62 & 56 & 0 & \multicolumn{2}{|c|}{120298} & 1727 \\
\hline & & 44,84 & 8,92 & 57,77 & 0,78 & 1,01 & 1,85 & 1,67 & 0,00 & 3,58 & 8,89 & 71,79 \\
\hline \multirow{2}{*}{ AUXILIAR } & 20,90 & 2358 & 472 & 2830 & 166 & 364 & 172 & 320 & 8 & 72 & 1102 & 3932 \\
\hline & & 44,74 & 6,60 & 54,29 & 2,54 & 5,58 & 2,64 & 4,90 & 0,12 & 1,103 & 16,89 & 80,34 \\
\hline \multirow{2}{*}{ ATENDENTE } & 5,20 & 586 & 142 & 728 & 94 & 85 & 0 & 142 & 1 & \multicolumn{2}{|c|}{$30 \mid 352$} & 1080 \\
\hline & & 44,66 & 8,09 & 56,36 & 6,08 & 5,50 & 0,00 & 9,18 & 0,06 & 1,94 & 22,77 & 91,96 \\
\hline \multirow{2}{*}{ TOTAL } & \multirow{2}{*}{36,10} & 4074 & 913 & 4987 & 286 & 483 & 234 & 518 & 9 & 222 & 1752 & 6739 \\
\hline & & 44,76 & 7,44 & 55,53 & 2,50 & 4,23 & 2,05 & 4,53 & 0,08 & 1,94 & 15,34 & 79,39 \\
\hline
\end{tabular}

\section{RESULTADOS}

A adoção do SCP, segundo o instrumento de ocupação média de $77 \%$. 0 perfil desses pacientes PERROCA ${ }^{15}$, demonstrou que na clínica em estudo, mostrou-se como necessitando de assistência durante um período de 16 dias, não houve pacientes intensiva, semi-intensiva e intermediária, nas de cuidados mínimos. Em média estão internados proporções demonstradas no Quadro 2. nessa unidade 14,6 pacientes, representando uma

\begin{tabular}{|c|c|c|c|c|c|c|c|c|c|c|c|c|c|c|c|c|c|c|c|}
\hline Assistência & 1 & 2 & 3 & 4 & 5 & 6 & 7 & 8 & 9 & 10 & 11 & 12 & 13 & 14 & 15 & 16 & TOTAL & MEDI A & DESVIO \\
\hline Intensiva & 2 & 2 & 5 & 4 & 4 & 4 & 3 & 4 & 3 & 5 & 4 & 4 & 4 & 4 & 3 & 4 & 59 & 3,7 & 0,9 \\
\hline Semi-intensiv & 3 & 3 & 1 & 5 & 6 & 5 & 4 & 4 & 4 & 3 & 4 & 8 & 8 & 3 & 4 & 3 & 68 & 4,3 & 1,8 \\
\hline Intermediária & 6 & 8 & 9 & 5 & 3 & 4 & 5 & 5 & 7 & 7 & 8 & 6 & 6 & 11 & 8 & 9 & 107 & 6,7 & 2,1 \\
\hline TOTAL & 11 & 13 & 15 & 14 & 13 & 13 & 112 & 13 & 14 & 15 & 16 & 18 & 18 & 18 & 15 & 16 & 234 & 14,6 & 2,2 \\
\hline
\end{tabular}

QUADRO 2 - Classificação dos pacientes segundo o tipo de assistência.

Os índices de ausências previstas e não previstas, segundo a categoria profissional, estão demonstrados no Quadro 3. 
QUADRO 3 - Percentual de ausências previstas e não previstas, segundo GAIDZINKI 8 .

\section{ENFERMEIROS}

\begin{tabular}{|l|l|l|l|l|l|l|l|l|}
\hline $\mathrm{e}$ & $(1+\mathrm{e})$ & $\mathrm{f}$ & $(1+\mathrm{f})$ & venfer & $(1+$ venfer $)$ & aenfer & $(1+$ Aenfer $)$ & $\left(1+\mathrm{D}^{\text {enter }}\right)$ \\
\hline 2 & 1,4 & 13 & 1,04 & 30 & 1,09 & 15 & 1,04 & 1,65 \\
\hline & & & & & & & & \\
\hline
\end{tabular}

\section{AUXILIARES/TÉCNICOS}

\begin{tabular}{|l|l|l|l|l|l|l|l|l|}
\hline e & $(1+\mathrm{e})$ & $\mathrm{f}$ & $(1+\mathrm{f})$ & Aux/téc & $(1+$ Vaux/téc $)$ & Aaux/ Téc & $(1+$ Aaux/ Téc $)$ & $\left(1+\mathrm{D}^{\text {enter }}\right)$ \\
\hline 2 & 1,4 & 13 & 1,04 & 30 & 1,09 & 15 & 0,04 & 1,65 \\
\hline & & & & & & & & \\
\hline
\end{tabular}

Foram utilizadas as letras E para designação dos índices de ausência relacionados ao descanso remunerado semanal, $\mathrm{F}$ para os índices referentes aos feriados não coincidentes com o Domingo, V para os indices referentes aos dias de férias e Apara os dias de cobertura das ausências não previstas (absenteísmo). $\mathrm{O}$ valor médio de 15 dias por ano de absenteísmo para enfermeiros (a/enf.) e de 20 dias por ano de absenteísmo para auxiliares de enfermagem (a/aux.) está fundamentado nos estudos de GAIDZINSKI et al. 10 desenvolvidos em hospitais públicos.

Não foram utilizados os índices de absenteísmo da unidade, demostrados no Quadro 1, para projeção do quadro de pessoal, por serem muito elevados (aproximadamente 9\% para enfermeiros e 17\% para auxiliares de enfermagem), sendo necessário um estudo mais aprofundado, objetivando estudar as causas das distorções que os elementos da equipe demonstraram.

Mediante os dados levantados procedeu-se à projeção do dimensionamento do pessoal de enfermagem da unidade pela aplicação da equação proposta por GAIDZINSKI 8. Os resultados estão apresentados no Quadro 4.

QUADRO 4- Projeção de quadro de pessoal de enfermagem, segundo a Resolução COFEN n 189/96.

\begin{tabular}{|c|c|c|c|c|c|c|c|c|c|c|c|c|c|}
\hline \multirow{2}{*}{ Assistência } & \multirow{2}{*}{$\mathrm{n}$} & \multirow{2}{*}{$\mathrm{h}$} & \multicolumn{2}{|c|}{ DISTRIBUIÇÃO } & \multicolumn{2}{|c|}{ CARGA DIÁRIA } & \multirow[b]{2}{*}{ t.p } & \multicolumn{2}{|c|}{ PESSOAL/DIA } & \multicolumn{2}{|c|}{ AUSÊNCIAS } & \multicolumn{2}{|c|}{ QUADRO PESSOAL } \\
\hline & & & Penf & Paux/téc. & Ent & Aux/Téd & & Enf & Aux/Ted & Enf & Aux/Tec & \begin{tabular}{l|l} 
Enf \\
\end{tabular} & Aux/Tec \\
\hline Intensiva & 3,7 & 15 & 56 & 44,4 & 31,68 & 25,30 & 6,4 & 4,95 & 3,95 & 1,65 & 1,67 & $8,, 17$ & 6,60 \\
\hline Semi-intensiva & 4,3 & 8,5 & 40 & 60 & 14,62 & 21,93 & 6,4 & 2,28 & 3,43 & 1,65 & 1,67 & 3,77 & 5,72 \\
\hline Intermediária & 6,7 & 4,9 & 27 & 73 & 8,86 & 23,97 & 6,4 & 1,39 & 3,74 & 1,65 & 1,67 & 2,29 & 6,25 \\
\hline SOMA & 15 & & & & 55,16 & 71,2 & & 8,62 & 11,12 & & & 15,00 & 19,00 \\
\hline \multicolumn{9}{|c|}{ TOTAL } & 19,74 & & & & 34,00 \\
\hline
\end{tabular}

O Quadro 4 demonstra que a carga diária de trabalho da clínica corresponde a 55,16 horas de enfermeiros e 71,20 horas de auxiliares de enfermagem.

Considerou-se que cada elemento da equipe trabalha, em média, 8h/dia com uma carga semanal de 40 horas semanais e com uma produtividade média de $80 \%$, ou seja, de dedicação exclusiva ao trabalho propriamente dito, isso representa (t.p) igual a 6,4 horas de trabalho efetivo por elemento da equipe de enfermagem.

As pessoas não são produtivas, igualmente, em todo o tempo do turno de trabalho, pois executam uma série de atividades não relacionadas às suas tarefas profissionais, como o atendimento de suas necessidades fisiológicas, períodos de descanso, trocas de informações não ligadas ao trabalho, deslocamentos necessários, comemorações e outras mais.

Segundo BISENG ${ }^{3}$, indices de produtividade entre $75 \%$ a $85 \%$ são considerados excelentes. Portanto, no presente estudo, tomou-se como valor médio $80 \%$ das horas de dedicação exclusiva ao trabalho.

Seguindo-se esse raciocínio, para atender ao perfil dos pacientes internados nessa unidade, segundo. o parâmetro horas de assistência de enfermagem por tipo de assistência da Resolução COFEN n¹89/966, necessita-se de um quadro de 34 pessoas, sendo 15 enfermeiros e 19 auxiliares de enfermagem.

Finalmente, no Quadro 5 apresenta-se a comparação do quadro projetado pela Resolução COFEN n $189 / 96^{6}$ com o quadro existente na unidade. 
Partindo-se da premissa, que o quadro de pessoal efetivo, no dia-a-dia, representa o total de pessoas subtraido do percentual médio de ausências proje existentes na unidade, tem-se que, diariamente, a menores, mais compativeis com a realidade, uma vez composição da equipe de enfermagem é, prati- que, uma projeção de quadro não pode estar camente, igual tanto no número de enfermeiros fundamentada em dados distorcidos, sendo necessário quanto no de auxiliares de enfermagem.
No entanto, o total geral difere um pouco do antes equacionar os problemas internos à unidade.

QUADRO 5- Comparação do quadro projetado pela Re unidade. solução COFEN n $189 / 96^{6}$ com o quadro existente na

\begin{tabular}{|c|r|r|r|r|r|r|}
\hline \multirow{2}{*}{ CATEGORIA } & QUANTIDADE DIARIA & \multicolumn{2}{|r|}{ TAXA DE AUSENCIAS } & \multicolumn{2}{|r|}{ QUANTIDADE MENSAL } \\
\cline { 2 - 7 } & EXISTENTE & PROJETADA & EXISTENTE & PROJETADA & EXISTENTE & PROJETADA \\
\hline ENFERMEIRO & 9,31 & 9,1 & 71,8 & 65 & 16 & 15 \\
\hline AUXILIARES & 11,64 & 11,4 & 80,3 & 67 & 21 & 19 \\
\hline TOTAL & 20,96 & 20,47 & & & 37,00 & 34,00 \\
\hline & 21,00 & 21,00 & & & & \\
\hline
\end{tabular}

\section{CONCLUSÃO}

O estudo relativo ao dimensionamento de pessoal de enfermagem permitiu concluir que o quadro existente na clínica respeita e até supera, discretamente, o preconizado pela Resolução COFEN $n^{\circ} 189 / 966$.

No entanto, a equipe de enfermagem, freqüentemente, refere que existe grande sobrecarga de trabalho, tendo a percepção de que o quadro de pessoal da unidade é menor do que o necessário. Essa talvez possa ser uma das justificativas para o alto indice de absenteísmo.

A percepção do quadro insuficiente, embora dentro dos parâmetros preconizados, indica a necessidade da realização de estudos mais aprofundados, referentes às horas de assistência de enfermagem, segundo a complexidade assistencial dos pacientes internados em uma unidade especializada em transplante hepático.

\section{REFERÊNCIAS BIBLIOGRÁFICAS}

1.ALCALÁ, M.U. et al. Cálculo de pessoal: estudo preliminar para o estabelecimento de pessoal de enfermagem na superintendência médico hospitalar de urgência. São Paulo, Secretaria de Higiene e Saúde, 1982.

2.ALVES, S.M. et al. Enfermagem: contribuição para um cálculo de recursos humanos na área. Rio de Janeiro, Coordenadoria de Comunicação Social do INAMPS , 1988.

3.BISENG, W. Administração financeira em engenharia clínica. São Paulo, 1996. /Workshop/.

4.CAMPEDELLI, M.C. et al. Cálculo de pessoal de enfermagem: competência da enfermeira. Rev.Esc.Enf.USP , v.21, n.1, p.3-15, 1987.

5.CHIZZOTTI, A. Pesquisa em ciências humanas e sociais. São Paulo, Cortez, 1991.

6.CONSELHO FEDERAL DE ENFERMAGEM. Resolução n ${ }^{\circ} 189 /$ 96. Estabelece parâmetros para dimensionamento do quadro de profissionais de enfermagem nas instituições de saúde. In: CONSELHO REGIONAL DE ENFERMAGEM. Documentos básicos de enfermagem: enfermeiros, técnicos e auxiliares. São Paulo, 1997. p.177-80.
7.FUGULIN, F.M.T.; GAIDZINSKI, R.R. Sistema de classificação de pacientes: análise das horas de assistência de enfermagem. Nursing, n.11, p.27-34, 1999.

8.GAIDZINSKI, R.R. Dimensionamento de pessoal de enfermagem em instituições hospitalares. São Paulo, 1998. 118p. Tese (Livre-Docência) - Escola de Enfermagem, Universidade de São Paulo.

9.GAIDZINSKI, R.R. Dimensionamento do pessoal de enfermagem segundo a percepção de enfermeiras que vivenciam essa prática. São Paulo, 1994. 259p. Tese (Doutorado) - Escola de Enfermagem, Universidade de São Paulo.

10.GAIDZINSKI, R.R. et al. Estudo das ausências da equipe de enfermagem num hospital geral de grande porte. Rev.Enf.Complexo HC/FMUSP, v.1, n.4, p.8-14, 1998.

11.GIOVANNETTI, P. Understanding patiente classification systems. J.Nurs.Adm., v. 9, n. 2, p. 4-9, 1979.

12.KURCGANT, P. et al. Subsídios para a estimativa de pessoal de enfermagem. Enfoque, v. 17, n. 3 , p. 79-81, 1989.

13.LIMA, M.L.A. Dimensionamento de pessoal de enfermagem em unidades de clínica médica: estudo exploratório. Rev. Gaúcha Enf., v. 6, n. 1, p. 125-42, 1985.

14.MAGAlHÃES, A.M.M.; DUARTE, E.R.M.; G.M.S.S. de. Estudo das variáveis que participam do dimensionamento de pessoal de enfermagem em hospitais de grande porte. Rev. Gaúcha Enf., v.16, n.1/2, p.5-16, 1995.

15.PERROCA, M.G. Sistema de classificação de pacientes: construção e validação de um instrumento. São Paulo, 1996. Dissertação (Mestrado) - Escola de Enfermagem, Universidade de São Paulo.

16.RIBEIRO, C.M. Sistema de classificação de pacientes como subsidios para provimento de pessoal de enfermagem. São Paulo, 1972. 98p. Tese (Doutorado) - Escola de Enfermagem, Universidade de São Paulo.

17.RODRIGUES FILHO, J. Sistema de classificação de pacientes parte I: dimensionamento de pessoal de enfermagem. Rev.Esc.Enf.USP, v.26, n.3, p.395-404, 1992.

18.SANTOS, S.R. Cálculos de pessoal de enfermagem: estudo de dois métodos. Rev.Esc.Enf.USP, v.26, n.2, p.137 - 54, 1992. 